\title{
Video Article \\ Basophil Activation Test for Investigation of IgE-Mediated Mechanisms in Drug Hypersensitivity
}

\author{
Markus Steiner ${ }^{1}$, Andrea Harrer ${ }^{2}$, Roland Lang ${ }^{3}$, Michael Schneider ${ }^{4}$, Fátima Ferreira ${ }^{5}$, Thomas Hawranek ${ }^{3}$, Martin Himly ${ }^{1}$ \\ ${ }^{1}$ Department of Molecular Biology, University of Salzburg \\ ${ }^{2}$ Department of Neurology, Paracelsus Medical University \\ ${ }^{3}$ Department of Dermatology, Paracelsus Medical University \\ ${ }^{4}$ Bühlmann Laboratories \\ ${ }^{5}$ Christian Doppler Laboratory for Allergy Diagnosis and Therapy, University of Salzburg
}

Correspondence to: Martin Himly at martin.himly@sbg.ac.at

URL: https://www.jove.com/video/3263

DOI: doi:10.3791/3263

Keywords: Immunology, Issue 55, NSAIDs, hypersensitivity, propyphenazone, diclofenac, drug conjugates, basophil activation test

Date Published: 9/16/2011

Citation: Steiner, M., Harrer, A., Lang, R., Schneider, M., Ferreira, F., Hawranek, T., Himly, M. Basophil Activation Test for Investigation of IgEMediated Mechanisms in Drug Hypersensitivity. J. Vis. Exp. (55), e3263, doi:10.3791/3263 (2011).

\section{Abstract}

Hypersensitivity reactions against non-steroidal anti-inflammatory drugs (NSAIDs) like propyphenazone (PP) and diclofenac (DF) can manifest as Type I-like allergic reactions ${ }^{1}$. In clinical practice, diagnosis of drug hypersensitivity is mainly performed by patient history, as skin testing is not reliable and oral provocation testing bears life-threatening risks for the patient ${ }^{2}$. Hence, evidence for an underlying IgE-mediated pathomechanism is hard to obtain.

Here, we present an in vitro method based on the use of human basophils derived from drug-hypersensitive patients that mimics the allergic effector reaction in vivo. As basophils of drug-allergic patients carry IgE molecules specific for the culprit drug, they become activated upon $\mathrm{IgE}$ receptor crosslinking and release allergic effector molecules. The activation of basophils can be monitored by the determination of the upregulation of CD63 surface expression using flow cytometry ${ }^{3}$.

In the case of low molecular weight drugs, conjugates are designed to enable IgE receptor crosslinking on basophils. As depicted in Figure 1 , two representatives of NSAIDs, PP and DF, are covalently bound to human serum albumin (HSA) via a carboxyl group reacting with the primary amino group of lysine residues. DF carries an intrinsic carboxyl group and, thus, can be used directly ${ }^{4}$, whereas a carboxyl group-containing derivative of PP had to be organochemically synthesized prior to the study ${ }^{1}$.

The coupling degree of the low molecular weight compounds on the protein carrier molecule and their spatial distribution is important to guarantee crosslinking of two IgE receptor molecules. The here described protocol applies high performance-size exclusion chromatography (HPSEC) equipped with a sequential refractive index (RI) and ultra violet (UV) detection system for determination of the coupling degree.

As the described methodology may be applied for other drugs, the basophil activation test (BAT) bears the potential to be used for the determination of IgE-mediated mechanisms in drug hypersensitivity. Here, we determine PP hypersensitivity as IgE-mediated and DF hypersensitivity as non-lgE-mediated by BAT.

\section{Video Link}

The video component of this article can be found at https://www.jove.com/video/3263/

\section{Protocol}

\section{Preparation of drug conjugates}

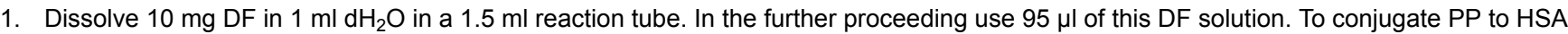
dissolve $30.4 \mathrm{mg}$ PP derivative $(304 \mathrm{~g} / \mathrm{mol})$ in $500 \mu \mathrm{l} 0.3 \mathrm{M}$ sodium hydroxide $(\mathrm{NaOH})$.

2. Add $430.6 \mu \mathrm{dH}_{2} \mathrm{O}$ and $100 \mu \mathrm{l} 0.5 \mathrm{M}$ 2-(N-morpholino)ethanesulfonic acid (MES) buffer $\mathrm{pH} 6.5$ to $95 \mu \mathrm{l}$ of dissolved DF. Add $33.1 \mu \mathrm{ld} \mathrm{H}_{2} \mathrm{O}$ and $140 \mu \mathrm{l}$ of $2 \mathrm{M}$ MES buffer $\mathrm{pH} 2.9$ to the PP sample.

3. Add $57.5 \mu \mathrm{l}$ of a freshly prepared $\mathrm{N}$-ethyl- $N$ '-(3-dimethylaminopropyl)carbodiimide hydrochloride (EDC) stock solution dissolved in $\mathrm{dH}_{2} \mathrm{O}$ with a concentration of $100 \mathrm{mg} / \mathrm{ml}$ to the DF sample, or $10 \mu \mathrm{l} \mathrm{EDC} \mathrm{solution} \mathrm{to} \mathrm{the} \mathrm{PP} \mathrm{sample.} \mathrm{Vortex} \mathrm{the} \mathrm{samples} \mathrm{for} 1$ minute.

4. Add $16.9 \mu \mathrm{l}$ of HSA solution with a concentration of $118 \mathrm{mg} / \mathrm{ml}$ to each sample.

5. Incubate the samples for 2 hours at room temperature while shaking at 300 rounds $/ \mathrm{min}$

6. Dialyze the DF and PP samples 3 times against 2 liters of phosphate-buffered saline (PBS) $\mathrm{pH} 7.4$ for several hours each. Perform all dialyzing steps at $4^{\circ} \mathrm{C}$. 
7. Centrifuge the sample 10 minutes at $14,000 \times g$ and collect the supernatant containing DF or PP conjugated to HSA.

8. Check supernatant for proteins by performing a SDS-PAGE, using $12 \%$ gels. Load about $12 \mu \mathrm{g}$ of HSA conjugate into one gel slot.

\section{Determine coupling rate of drug conjugates (Figure 2)}

1. For the analysis of the coupling rate of DF and PP conjugates by HPSEC use a $100 \mathrm{mM}$ sodium phosphate buffer $\mathrm{pH} 6.5 \mathrm{containing} 150 \mathrm{mM}$ $\mathrm{NaCl}$ and $0.05 \%$ sodium azide. Use a $7.8 \times 300 \mathrm{~mm}$ TSK-Gel-G2000 $\mathrm{swxL}$ column.

2. Perform detection of signals by using an online-coupled $\mathrm{RI}$ and UV detector system.

3. Prepare a HSA standard sample with a concentration of $1 \mathrm{mg} / \mathrm{ml}$ in $\mathrm{dH}_{2} \mathrm{O}$. For detector calibration inject $50 \mu \mathrm{HSA}$ standard solution.

4. Calculate the RI constant $\mathrm{k}_{\mathrm{RI}}$ and the UV constant $\mathrm{k}_{\mathrm{UV}}$ for the detector. Use the formulas are $a_{\mathrm{Rl}}=\mathrm{k}_{\mathrm{RI}}{ }^{*}\left[(\mathrm{dn} / \mathrm{dc})_{\mathrm{HSA}}{ }^{*} \mathrm{C}(\mathrm{HSA})\right]$ and area $a_{U V}=\mathrm{k}_{U V}{ }^{*}[(\mathrm{dA} /$ $\left.\mathrm{dc})_{\mathrm{HSA}}{ }^{*} \mathrm{C}(\mathrm{HSA})\right]$ with $(\mathrm{dn} / \mathrm{dc})_{\mathrm{HSA}}=0.185$ and $(\mathrm{dA} / \mathrm{dc})_{\mathrm{HSA}}=0.518^{5}$.

5. Prepare DF and PP derivative standards for HPSEC that 5,10 , and $30 \mathrm{nmol}$ amounts of both standards can be easily injected.

6. Calculate mean values for $(\mathrm{dn} / \mathrm{dc})_{\text {drug }}$ and $(\mathrm{dA} / \mathrm{dc})_{\text {drug }}$ for both standards. For $\mathrm{DF}$, a $(\mathrm{dn} / \mathrm{dc})$ of $0.235 \pm 0.010$ and a (dA/dc) of $39.000 \pm 0.493$ was determined. For PP, a (dn/dc) of $0.328 \pm 0.016$ and a $(\mathrm{dA} / \mathrm{dc})$ of $53.155 \pm 2.464$ was determined.

7. Inject drug conjugates on HPSEC and determine their RI and UV peak areas.

8. Calculate the concentration of DF, PP derivative, and HSA by solving the two equations area ${ }_{\mathrm{Rl}}=\mathrm{k}_{\mathrm{RI}}{ }^{*}\left[(\mathrm{dn} / \mathrm{dc})_{\mathrm{drug}}{ }^{*} \mathrm{C}(\mathrm{drug})+(\mathrm{dn} / \mathrm{dc})_{\mathrm{HSA}}{ }^{*} \mathrm{C}(\mathrm{HSA})\right]$ and area ${ }_{U V}=k_{U V}{ }^{*}\left[(d A / d c)_{d r u g}{ }^{*} \mathrm{c}(\right.$ drug $\left.)+(d A / d c)_{H S A}{ }^{*} \mathrm{c}(H S A)\right]$ for the unknowns.

\section{BAT using drug conjugates}

1. Prepare seven flow cytometry vials and label them depending on their content: unstained control, negative control, positive control, and drug conjugates.

2. Pipette $50 \mu \mathrm{l}$ of B-CCR-STB stimulation buffer (Flow2 CAST, Bühlmann Laboratories, Schönenbuch, $\mathrm{CH}$ ) into the vials reserved for the unstained and negative control. As a positive control, use $50 \mu \mathrm{l}$ of an anti-FcERI solution provided by the Flow2 CAST kit. Add the appropriate amounts of conjugate solution to the vials reserved for the drug conjugates by creating a 1:10 dilution series from $0.02-20 \mu \mathrm{g} / \mathrm{ml} \mathrm{DF}$ conjugate and PP conjugate (final concentrations in an assay volume of $200 \mu \mathrm{l}$ ). These titration experiments must be performed to determine the concentration of optimal basophil activation for each patient sample.

3. Add $100 \mu \mathrm{l}$ stimulation buffer to each tube and $50 \mu \mathrm{l}$ patient's EDTA whole blood. Mix the samples carefully.

4. Pipette $20 \mu$ l Flow2 CAST staining reagent containing anti-CCR3 antibodies labeled with phycoerythrin (PE) and anti-CD63 antibodies labeled with fluorescein isothiocyanate (FITC) to each tube and mix again. Do not pipette staining reagent into the unstained sample!

5. Incubate the samples for 45 minutes in a $37^{\circ} \mathrm{C}$ water bath.

6. Stop reaction on ice for 5 minutes.

7. Lyse erythrocytes by adding $2.0 \mathrm{ml}$ Flow2 CAST lysing reagent to each vial and vortex gently. Incubate samples in the dark for 10 minutes at room temperature.

8. Centrifuge samples for 5 minutes at $500 \times \mathrm{g}$ and carefully decant supernatant.

9. Resuspend cells in $500 \mu \mathrm{l}$ Flow2 CAST wash buffer and store on ice until flow cytometry.

10. Adjust the flow cytometer's voltage settings for forward and side scatter (FSC, SSC) while acquiring the unstained sample.

11. Gate the cells predicted as lymphocytes from the SSC-FSC plot to the SSC-PE plot. Basophils are identified by a PE-labeled anti-CCR3 antibody as $\mathrm{CCR}^{\mathrm{hi}} \mathrm{SSC}^{\mathrm{lo}}$ and gate them into the FITC-PE plot. The anti-CD63 antibody detecting activated basophils is labeled with FITC.

12. Set the cut-off to maximum $2.5 \%$ of basophils as $\mathrm{CD} 63^{\mathrm{hi}}$ in the negative control. A sample is considered positive for basophil activation if $>5 \%$ basophils are $\mathrm{CD}_{6} 3^{\mathrm{hi}}$ and the mean fluorescence index (MFI) of the sample divided by the MFI of the negative control exceeds 2 (Figure 3 ).

\section{Representative Results:}

This experiment evaluates BAT as a beneficial tool to detect IgE-dependent drug hypersensitivity. Protein conjugates of NSAIDs are used for activation of basophils. By HPSEC with RI and UV detection aggregation state, coupling degree, and effective yield of the conjugates are determined. As Figure 4 shows, $43.5 \%$ of the DF conjugates remained monomeric with a coupling degree of 5.0 DF/HSA. For the aggregates a coupling degree of 9.5 was determined. The propyphenazone conjugate was shown to consist of $68.5 \%$ monomers with a coupling degree of 21.2. The coupling degree of the aggregates was 27.9. In total, the determined coupling degree of DF conjugates was 7.6 DF and that of PP conjugates was 23.2 .

BAT performed with conjugates under optimized conditions (concentration of conjugates, stimulation time) allowed investigation of lgEdependent reactions in NSAID hypersensitivity. As shown in Figure 5, only the PP conjugate was able to trigger basophil activation visualized by an upregulation in CD63 surface expression: $20.6 \%$ of basophils were activated characterized by a log-shift in fluorescence intensity. The MFI increased by a factor of 4.9 from 386 (negative control) to 1893. In contrast, using the DF conjugate only $3.1 \%$ of the basophils were activated which was below the 5\% cut-off. Also the MFI increased only by a factor 1.1 (limit 2.0) from 197 (negative control) to 210 . Assay validity is given by (i) $<5 \%$ basophil activation in the negative control, (ii) a log-shift in fluorescence intensity of a basophil subpopulation, and (iii) $>50 \%$ activated basophils (positive control). 
<smiles>O=C(O)Cc1ccccc1Nc1c(Cl)cccc1Cl</smiles>

\section{PP}<smiles>Cc1c(C(C)C)c(=O)n(-c2ccccc2)n1CCCC(=O)O</smiles>
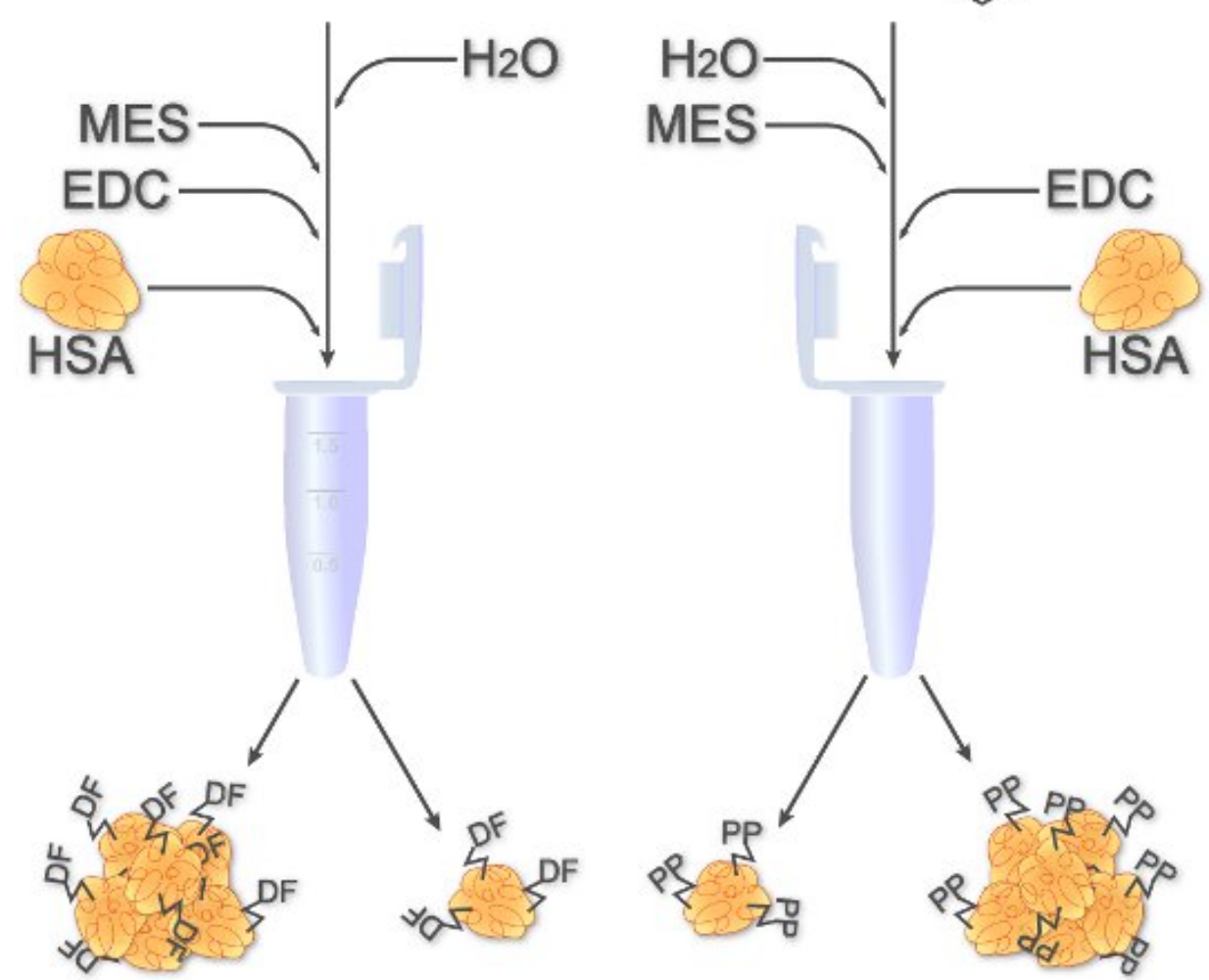

Figure 1. Coupling of DF and PP derivative to HSA. After dissolving the drugs, water, MES buffer, and EDC (coupling reagent) are added. The samples are vortexed for 1 minute before HSA is pipetted to the drug solutions. Within 2 hours shaking at room temperature the drugs covalently bind to HSA. Monomers as well as aggregates may result. 


\section{Calculate constants $\mathrm{K}_{\mathrm{R}}$ and $\mathrm{Kuv}_{\mathrm{v}}$}

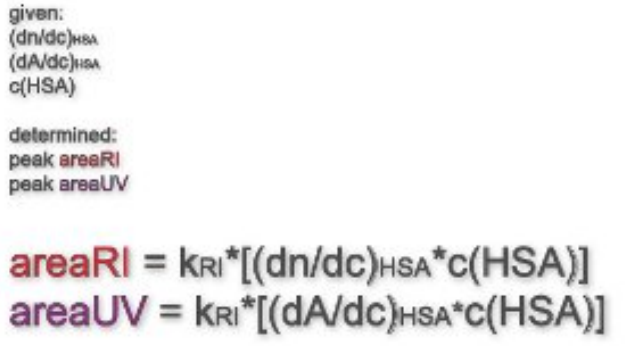

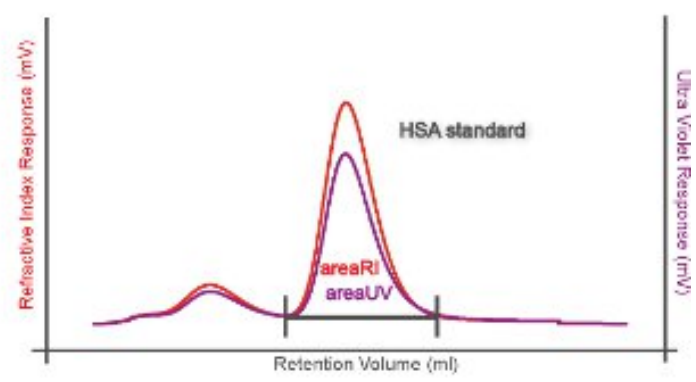

2. Calculate mean (dn/dc) drug and $(\mathrm{d} / \mathrm{d} / \mathrm{dc}))_{\mathrm{drug}}$

given:
kow
oddrug)

determined:

peak areaRI

peak areaUV

\section{areaRI $=\mathrm{kR}_{\mathrm{R}}[(\mathrm{dn} / \mathrm{dc})$ drug*c(drug)] \\ areaUV $=\operatorname{kuv} \cdot\left[(\mathrm{dA} / \mathrm{dc}) \mathrm{drug}^{*} \mathrm{c}(\mathrm{drug})\right]$}

\section{Calculate $c(d r u g)$ and $c(H S A)$}

given:
kRI
kUV
(dn/dc)enen
(dA/dc)enva
(dn/dc)msa
(dA/dc)mea
determined:
peak areaRI
peak areaUV

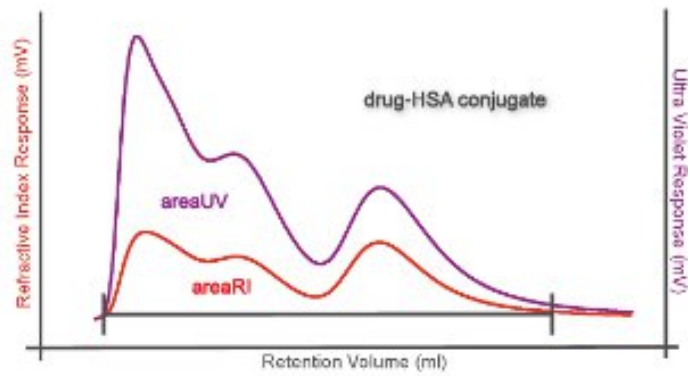

\section{areaRI $=\mathrm{kRI}^{*}\left[(\mathrm{dn} / \mathrm{dc})\right.$ drug $\left.{ }^{*} \mathrm{c}(\mathrm{drug})+(\mathrm{dn} / \mathrm{dc}) \mathrm{HSA}^{*} \mathrm{c}(\mathrm{HSA})\right]$ areaUV $=k u^{*}\left[(\mathrm{dA} / \mathrm{dc})\right.$ drug $\left.{ }^{*} \mathrm{c}(\mathrm{drug})+(\mathrm{dA} / \mathrm{dc}) \mathrm{HSA}^{*} \mathrm{c}(\mathrm{HSA})\right]$}

Figure 2. Calculation of coupling degrees. First, the constants $k_{R I}$ and $k_{U V}$ are determined. Therefore, HSA standard with known concentration is injected into the HPSEC system. $(\mathrm{dn} / \mathrm{dc})$ and $(\mathrm{dA} / \mathrm{dc})$ of HSA are given values of 0.185 and 0.518 , respectively ${ }^{5}$. The peak areas are used for calculating the constants. Second, for each drug three standards are injected to determine drug- and concentration-specific RI and UV areas. Since the $k_{R I}$ and $k_{U V}$ constants are known from the steps above, the drug-specific $(\mathrm{dn} / \mathrm{dc})$ and (dA/dc) derivatives can be calculated. Third, drug-HSA conjugates are injected into HPSEC. The resulting peak areas are used to calculate the concentrations of drug and HSA per peak by solving the two equations with two unknown variables.
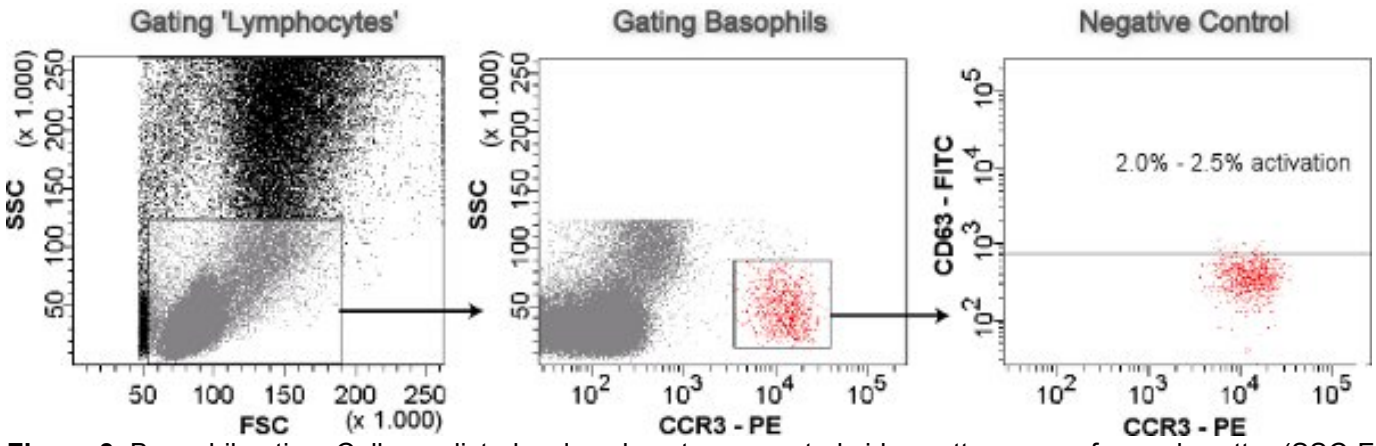

Figure 3. Basophil gating. Cells predicted as lymphocytes are gated side scatter versus forward scatter (SSC-FSC plot). The basophils are identified as CCR3 ${ }^{\text {hi }}$ SSC $^{l o}$ from the gated lymphocytes (SSC-CCR3-PE plot). Basophils are analyzed for activation (CD63 ${ }^{\text {hi }}$ ) in the CD63-FITCCCR3-PE plot. The negative control is used to set the cut-off of maximum $2.5 \%$ basophils remaining CD $63^{\mathrm{hi}}$. 


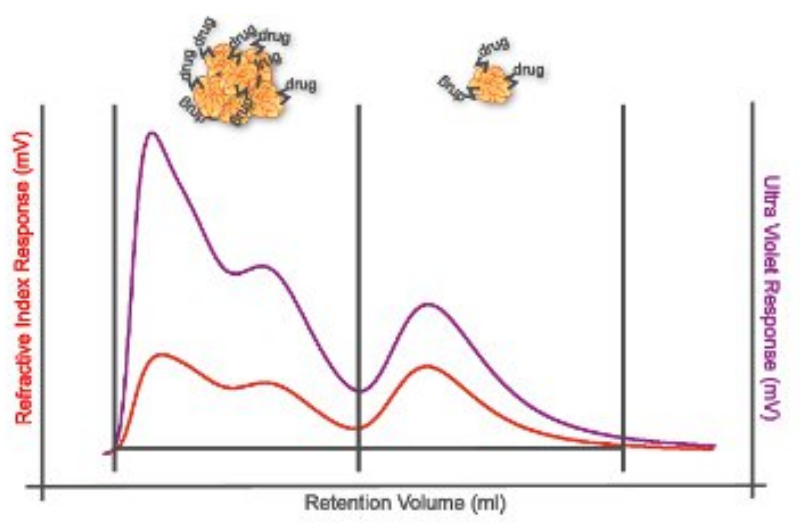

DF

$43.5 \%$ \%

5.0 DF/HSA

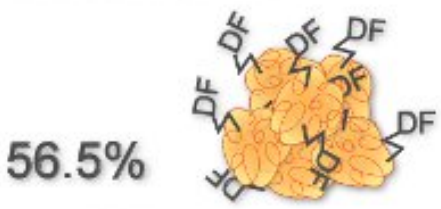

9.5 DF/HSA

PP

$68.5 \%$ PY

21.2 PP/HSA

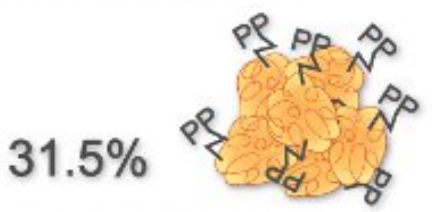

27.9 PP/HSA

\section{total 7.6 DF/HSA}

\section{total $23.2 \mathrm{PP} / \mathrm{HSA}$}

Figure 4. Determined coupling degrees of drug conjugates. RI and UV signals show two peaks representing aggregates (eluting at low retention volume) and monomers (eluting at high retention volume) of drug-HSA conjugates. Percentages of monomers and aggregates (determined from RI signals) and their coupling degrees are depicted $(n=1)$.
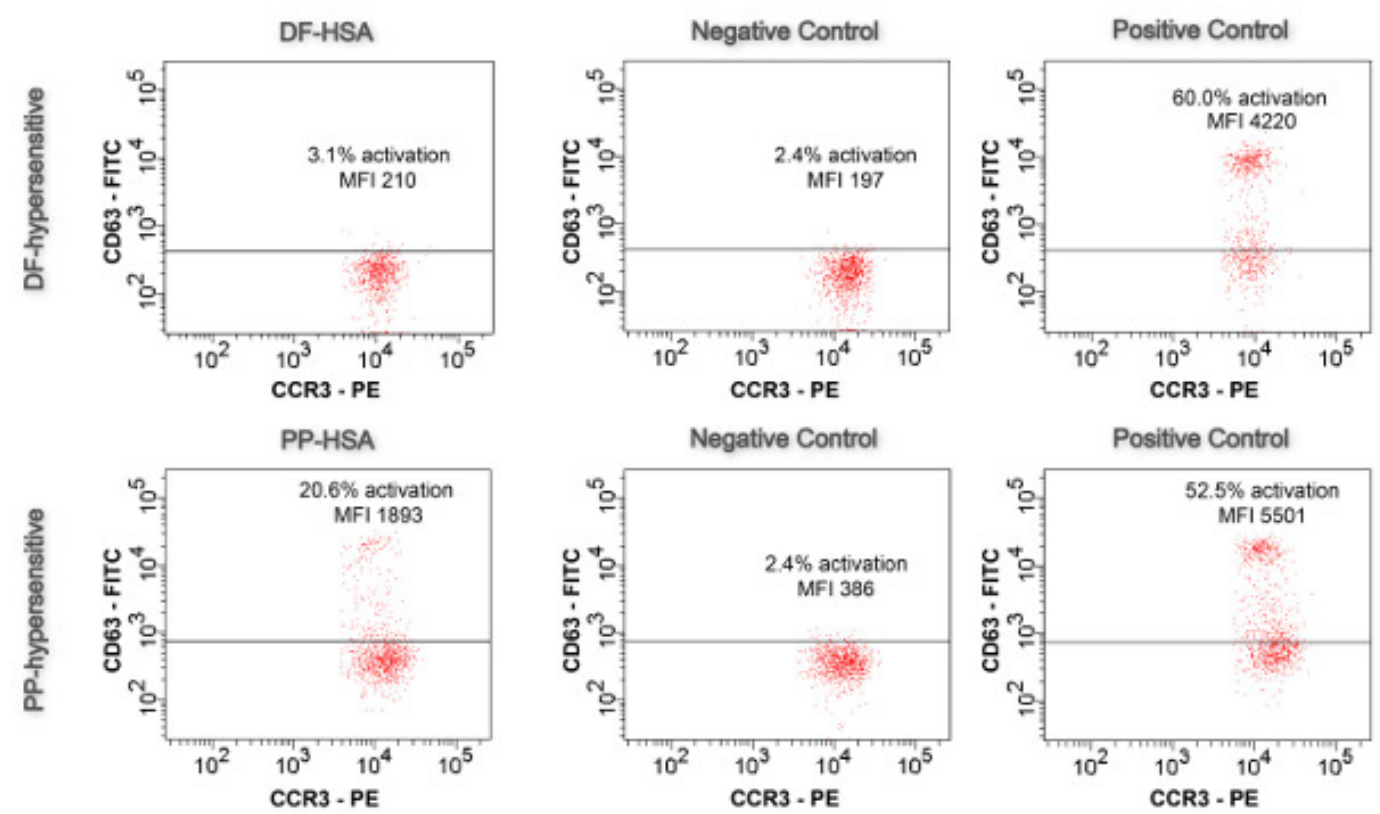
Figure 5. Basophil activation test. Results of drug conjugate-activated samples, negative controls, and positive controls are shown in CD63FITC-CCR3-PE plots $(n=1)$.

\section{Discussion}

BAT is a well-established although not yet routinely used method for diagnosis of IgE-mediated allergic disease ${ }^{6,7}$. For drug hypersensitivity, however, its applicability is compromised as low molecular weight compounds are not able to crosslink IgE receptors, a prerequisite for basophil activation ${ }^{8}$. Therefore, drugs under investigation need to be covalently coupled to suitable carrier proteins (e.g. HSA). Importantly, the coupling degree (i.e. number of drug molecules per carrier protein) needs to be controlled to guarantee immunological activity (i.e. IgE receptor crosslinking) of conjugates. Theoretically, two haptens per carrier molecule should be sufficient for IgE receptor cross-linking and as few as five DF molecules per HSA have been shown to trigger mediator release in a cell-based assay ${ }^{4}$. Both conjugates, PP-HSA and DF-HSA, have been determined to display a sufficiently high coupling degree (HPSEC) and to be immunologically active making them suitable reagents for use in BAT. Another issue may be that drug metabolites may play a role, as a metabolite instead of the parent drug may cause the hypersensitivity reaction. In case of DF, this possibility has been evaluated in detail previously using five major Phase I metabolites and one linkage variant ${ }^{4}$. Important factors for mastering the described technique include quality of blood sample $(<12$ hours since blood collection, number of detected basophils $>500$ ) and optimization of stimulation conditions (time, dose). In addition, so-called non-responders who do not even react with the positive control (antibody directed against the $\mathrm{IgE}$ receptor) have to be identified. Therefore, validation criteria have to include an antiFcERI antibody as positive control. An important advantage of using drug conjugates is the fact that they appear non-toxic in contrast to pure drugs, as shown for DF-HSA conjugate by co-stimulation with anti-FceRI antibody in BAT ${ }^{4}$. Pure DF, in contrast, displays cytotoxic effects at a concentration of $1.25 \mathrm{mg} / \mathrm{ml}$ causing problems with the interpretability of BAT ${ }^{9}$. Generally, testing for potential cytotoxicity is strongly recommended for every newly produced drug conjugate.

Here, we have shown the potential of PP-HSA conjugate used in BAT to reveal IgE-mediated hypersensitivity. In contrast, DF hypersensitivity is not associated with IgE demonstrated by the lack of basophil activation in response to DF-HSA conjugate. Importantly, in case of uncertainty concerning an IgE-mediated mechanism, conjugates have to be evaluated for immunological activity by in vitro cell-based assay systems as has been shown for $\mathrm{DF}^{4}$.

Using the described setup of conjugating low molecular weight drugs covalently to suitable protein carrier molecules a number of hypersensitivity reactions against drugs including antibiotics, other NSAIDs, radiocontrast media, muscle relaxants, anesthetics, etc. may be investigated for an involvement of an IgE-mediated mechanism. Hence, BAT may serve as an addition to the existing methods for diagnosis (skin test, oral provocation testing).

\section{Disclosures}

No conflicts of interest declared.

\section{Acknowledgements}

This work was funded by the Austrian Science Fund (FWF) grant P18820-B13.

\section{Ethics statement:}

The study was approved (PLUS_Ethik_090514) by the Ethics Committee for Experiments Involving Humans and/or Animals at the University of Salzburg complying with the Helsinki Declaraction as revised in 1983 and all patients participating gave their written informed consent.

\section{References}

1. Himly, M., et al. IgE-mediated immediate-type hypersensitivity to the pyrazolone drug propyphenazone. J Allergy Clin Immunol. 111, 882-888, doi:S009167490291482X [pii] (2003).

2. Demoly, P., Pichler, W., Pirmohamed, M. \& Romano, A. Important questions in Allergy: 1--drug allergy/hypersensitivity. Allergy. 63, 616-619, doi:ALL1693 [pii] 10.1111/j.1398-9995.2008.01693.x (2008).

3. Ebo, D.G., Hagendorens, M.M., Bridts, C.H., Schuerwegh, A.J., Clerck, L.S., \& Stevens, W.J. Flow cytometric analysis of in vitro activated basophils, specific IgE and skin tests in the diagnosis of pollen-associated food allergy. Cytometry Part B (Clinical Cytometry). 64B (2005).

4. Harrer, A., et al. Diclofenac hypersensitivity: antibody responses to the parent drug and relevant metabolites. PLoS One. 5, e13707, doi:10.1371/journal.pone.0013707 (2010).

5. Wen, J., Arakawa, T., \& Philo, J.S. Size-exclusion chromatography with on-line light-scattering, absorbance, and refractive index detectors for studying proteins and their interactions. Anal Biochem. 240, 155-166, doi:S0003-2697(96)90345-0 [pii] 10.1006/abio.1996.0345 (1996).

6. De Weck, A.L., Sanz, M.L., Gamboa, P.M., Aberer, W., Bienvenu, J., Blanca, M., Demoly, P., Ebo, D.G., Mayorga, L., Monneret, G., \& Sainte Laudy, J. Diagnostic tests based on human basophils: more potentials and perspectives than pitfalls. II. Technical issues. J Investig Allergol Clin Immunol. 18 (3), 143-155 (2008).

7. Ebo, D.G., et al. Flow-assisted allergy diagnosis: current applications and future perspectives. Allergy. 61, 1028-1039, doi:ALL1039 [pii] 10.1111/j.1398-9995.2006.01039.x (2006).

8. Poulsen, L.K., Quan, S., Kragh, C., Platzer, M.H., \& Skov, P.S. The basophil granulocyte in allergic reactions: experimental models and their use for the identification of drugs with effects or side effects on basophils. Curr Med Chem - Anti-inflammatory \& Anti-Allergy Agents. 3 (2), 167-180 (2004). 
9. Sanz, M.L., Gamboa, P., \& de Weck, A.L. A new combined test with flowcytometric basophil activation and determination of sulfidoleukotrienes is useful for in vitro diagnosis of hypersensitivity to aspirin and other nonsteroidal anti-inflammatory drugs. Int Arch Allergy Immunol. 136, 58-72, doi:IAA2005136001058 [pii] 10.1159/000082586 (2005). 